\title{
CHLAMYDIA TRACHOMATIS ANTIGEN DETECTION IN PREGNANCY AND ITS VERIFICATION BY ANTIBODY BLOCKING ASSAY
}

\author{
*R Malenie, PJ Joshi, MD Mathur
}

\begin{abstract}
Purpose: To detect the prevalence of genital infection caused by Chlamydia trachomatis in pregnant women and also to confirm the positive results using blocking antibody assay. Methods: Endocervical specimens were collected from 200 symptomatic and asymptomatic pregnant women attending the ANC OPD at M P Shah Medical College, Jamnagar. The samples were tested for presence of Chlamydia trachomatis antigen using the monoclonal antibody. Blocking antibody assay was used to further verify the positive results. Results: Out of 200 pregnant women, 38 (19\%) were found positive for Chlamydia trachomatis antigen. Out of the 68 symptomatic patients, $C$. trachomatis antigen was detected in $26.4 \%$. After verification of the positive samples $13.6 \%$ of the asymptomatic pregnant women were found to be harbouring the infection in their genital tract. Two $(5.2 \%)$ out of the 38 positive samples, on verification with the blocking antibody assay, were found to be false positive by IDEIA, ${ }^{\mathrm{TM}}$ thus the specificity of the IDEIA ${ }^{\mathrm{TM}}$ being $94.8 \%$. In patients with previous history of abortions, $27.7 \%$ were tested positive for $C$. trachomatis infection. Conclusions: Significant number of pregnant women shad $C$. trachomatis antigen in their endocervical canal, which can be easily diagnosed by this simple enzyme immuno assay having a specificity of $94.8 \%$. Verification of positive results by antibody blocking assay can further improve the specificity of this non-culture test. Asymptomatic patients should also be screened for the infection. History of previous abortions places the patient at a higher risk for $C$. trachomatis infection thus such patients should be definitely tested for chlamydia infection.
\end{abstract}

Key words: Chlamydia trachomatis, antigen, blocking antibody, pregnancy

C. trachomatis infection, a prevalent sexually transmitted disease, is associated with complications like ectopic pregnancy, fallopian tube block and adverse pregnancy outcome. ${ }^{1-4}$ In majority of the women the infection with this organism is asymptomatic or with minimal symptoms. Therefore, screening of women at risk is highly recommended.

Women at highest risk often have the least access to health care facilities. Therefore there is a need for a rapid, simple and accurate test to detect $C$. trachomatis infection, which can be performed outside the laboratory setting when the patient is still in the clinical setting.

Detection of $C$. trachomatis infection by non-culture techniques became feasible with the recent development of immunologic reagents specific for chlamydia. The direct fluorescent antibody (DFA) staining and enzyme immunoassay (EIA) are two such tests.

C. trachomatis is an infectious agent in pregnant women. Depending upon the population studied and the method used for diagnosis the prevalence ranges from $2-37 \%{ }^{5,6}$ Studies have

*Corresponding author (email: <malenie@ rediffmail.com>) Department of Microbiology (RM, MDM) Subharati Medical College, Meerut - 250 002. UP; and Department of Microbiology (PJJ), MP Shah Medical College, Jamnagar - 361 001, Gujarat, India Received : 06-05-05

Accepted : 06-12-05 proved a definite role of $C$. trachomatis infection in adverse pregnancy outcome. ${ }^{1-4}$ If not treated on time their newborns run a $20-40 \%$ risk of developing chlamydial conjunctivitis ${ }^{7-9}$ and a $10-20 \%$ risk of developing chlamydial pneumonia. ${ }^{7,9,10}$ Studies from India have reported that $15 \%$ of young asymptomatic women are positive for this infection. ${ }^{11}$ In this study, an effort was made to detect $C$. trachomatis antigen from the endocervical specimens of the pregnant women and to verify the positive results with the antibody blocking assay so as to achieve the most accurate results.

\section{Materials and Methods}

\section{Patients}

A total of 200 patients attending the prenatal clinic of M.P. Shah Medical College, Jamnagar, (India) were randomly included in our study. 68 had various symptoms like - vaginal discharge, dysuria and pruritis vulva while 132 were asymptomatic. A standard questionnaire including age, parity, abortions, educational status, socio-economic background, occupation, sexual behavior, occurrence of vaginal discharge, dysuria, pruritis, pain, ulcer warts, contraception and treatment if taken, was completed for every patient. The study was thoroughly explained to the patient and a written consent was obtained before taking any specimen. A complete gynaecological examination for lesions on external genitalia and a speculum examination for any cervical lesion or inflammation were done by the gynaecologist. 


\section{Clinical specimen}

The specimen was collected as per the instructions of the kit manufacturer. To obtain a satisfactory specimen from the cervix the patient was examined in lithotomy position and the cervix was visualized using a bivalve speculum and wiped with sterile gauze held on sponge forceps so as to remove excess mucus/blood/pus etc. Endocervix was swabbed using a dacron tip swab provided with the kit. The swab was inserted approximately $1 \mathrm{~cm}$ into the cervical canal and rotated several times before withdrawing. The swab was removed without touching the vaginal surface and placed in $1 \mathrm{~mL}$ working strength transport medium in a heat resistant vial. Specimens were immediately transferred to the laboratory where they were stored at $2-8^{\circ} \mathrm{C}$ for no longer than seven days prior to testing.

\section{Materials and Methods}

IDEIA ${ }^{\mathrm{TM}}$ PCE Chlamydia and IDEIA ${ }^{\mathrm{TM}}$ Chlamydia blocking reagent manufactured by DAKO Diagnostics Ltd. Cambridgeshire, UK were used. Former is an immunoassay using dual amplification technology for detection of chlamydia antigen in endocervical swabs and the latter is a blocking antibody reagent, which is to be used in conjunction for verification of positive reactions.

The IDEIA ${ }^{\mathrm{TM}}$ PCE Chlamydia blocking reagents consist of two reagents: a genus specific murine monoclonal antibody (blocking reagent) of different origin to that used in the IDEIA $^{\mathrm{TM}}$ PCE Chlamydia test and a murine monoclonal antibody with no anti-chlamydial activity (control reagent). The selective blocking test is performed on specimens found to be reactive in the IDEIA ${ }^{\mathrm{TM}}$ PCE Chlamydia test. Each reactive specimen is retested using two wells in the IDEIA ${ }^{\mathrm{TM}}$ PCE Chlamydia test. If Chlamydia LPS antigen is present in the specimen, the blocking antibody (but not the control antibody), will selectively bind to it and block the binding of Chlamydial LPS to the capture antibody on the coated well. On completion of the IDEIA ${ }^{\mathrm{TM}}$ PCE Chlamydia test procedure, a significant reduction in the absorbance value obtained in wells containing the specimen or positive control and blocking reagent, relative to the wells containing the specimen or positive control and control reagent verifies the presence of chlamydia LPS antigen. Adding 0.05 to the mean of the negative control values calculates the cut off value.

\section{Statistical analysis}

By using $\mathrm{Z}$ test for proportions $\mathrm{Z}$ (calculated) is 15.79 . At $5 \%$ and $1 \%$ level of significance $Z$ (tabulated) are 1.96 and 2.58 respectively. Since calculated value is greater than both the tabulated values, we reject the null hypothesis of no difference and significant difference between the results of two tests performed is accepted.

\section{Results}

Out of a total of 200 pregnant women included in the study group, 38 (19\%) were positive for Chlamydia antigen. Sixty-eight patients had discharge per vagina, dysuria, genital ulcer and cervicitis, out of which 18 (26.4\%) were positive for Chlamydia antigen (Fig. 1).

Cervicitis was the commonest finding (Table 1) associated with C.trachomatis infection (28.5\%).

Among the 132 asymptomatic pregnant women 20 (15.1\%) were positive for Chlamydia antigen (Fig. 1). Out of the 20 asymptomatic women $18(13.6 \%)$ were confirmed as positive with the blocking antibody assay while $2(5.2 \%)$ were found to be false positive. The highest incidence (33.3\%) of infection was found in the youngest of age group i.e, less than 20 years of age (Fig. 2).

History of previous abortions was present in 36 patients 32 of whom had spontaneous abortions and four underwent medical termination of pregnancy (MTP). Out of the patients with previous spontaneous abortion $27.7 \%$ were positive for

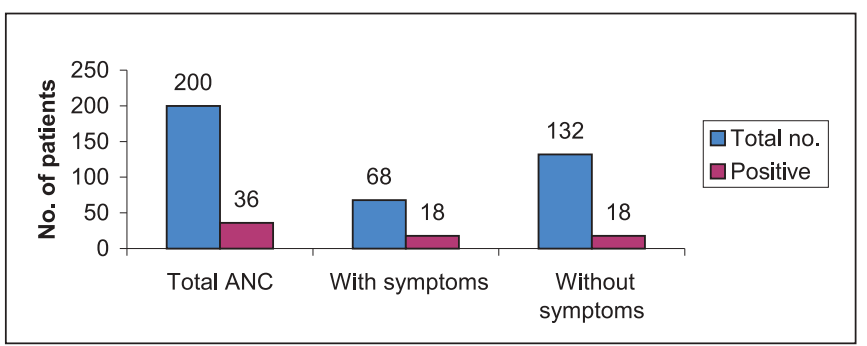

Figure 1: Incidence of $C$. trachomatis in pregnancy

\begin{tabular}{|c|c|c|c|}
\hline Signs/symptoms & $\begin{array}{c}\text { No. of } \\
\text { patients }\end{array}$ & $\begin{array}{c}\text { Positive } \\
\text { C. trachomatis }\end{array}$ & $\begin{array}{c}\text { Incidence } \\
\%\end{array}$ \\
\hline Vaginal discharge & 54 & 09 & 16.6 \\
\hline Dysuria & 26 & 05 & 18.2 \\
\hline Genital ulcer & 04 & 00 & 00 \\
\hline Cervicitis & 14 & 04 & 28.5 \\
\hline
\end{tabular}

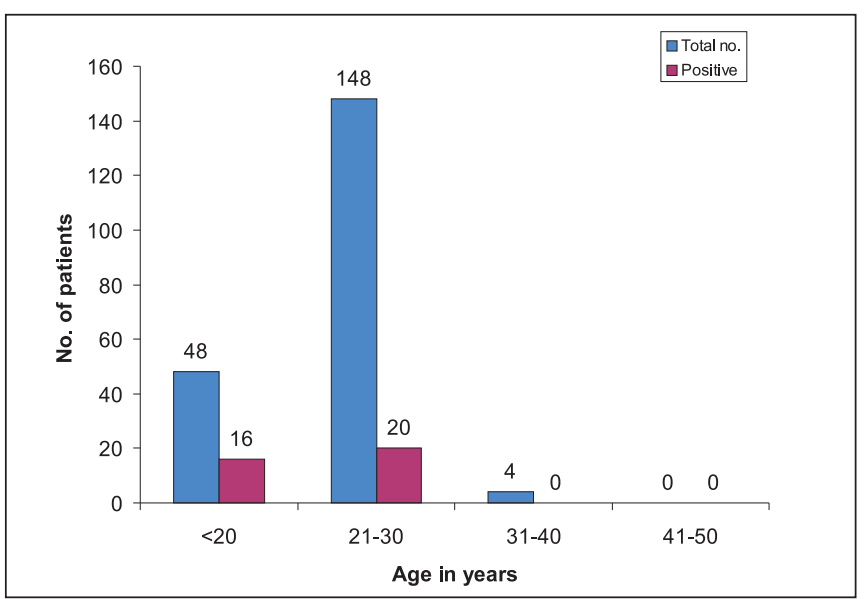

Figure 2: Incidence of C.trachomatis in pregnant women according to age 
Table 2: Association of $C$. trachomatis infection with previous abortions

\begin{tabular}{lccc}
\hline Type of abortion & Total no. & Positive & Incidence \% \\
\hline Spontaneous abortions & 32 & 08 & 25.0 \\
MTP & 04 & 02 & 50.0 \\
Total & $\mathbf{3 6}$ & $\mathbf{1 0}$ & $\mathbf{2 7 . 7}$ \\
\hline
\end{tabular}

MTP- medical termination of pregnancy

Chlamydia infection, while $50 \%$ of patients with previous MTP were positive (Table 2).

The 38 positive samples, which were positive with IDEIA, were re-confirmed by blocking antibody assay. Two (5.2\%) were found to be false positive thus the prevalence of Chlamydia infection in pregnancy in our study group was $18 \%$. All the discrepant results were from the asymptomatic group of pregnant women. Therefore, $13.6 \%$ of the asymptomatic group had chlamydial infection. The specificity of the EIA was $94.8 \%$.

\section{Discussion}

Various authors using different techniques have reported the incidence of $6-37 \%$ Chlamydia infection in pregnancy. ${ }^{3,11-16}$ An overall high incidence of Chlamydia antigen was detected in this study from the endocervical specimens, which was due to the study group selected. The study group comprised of patients of younger age belonging to poor socioeconomic group and included mostly the migratory population. Their partners were truck drivers/factory workers and defence personnel, thus placing their women at a higher risk of sexually transmitted diseases. Some reports of false positive Chlamydia antigen results could be because the patients who received pretreatment with antibiotics will remain asymptomatic although the antigen from the non viable Chlamydia can still be detected by EIA,,$^{15,18-19}$ while cultures are positive only if the organism is viable. Therefore, authors who used the cell culture technique to isolate Chlamydia from the endocervical specimen reported a low incidence. ${ }^{3}$

Quality of endocervical specimens collected can affect the incidence of false positive results because of its likely contamination with vaginal secretions..$^{20}$ EIA has been reported to give false positive results in presence of gram positive bacteria including Staphylococcus aureus, Streptococcus agalactiae, Peptostreptococcus spp. and gram negative organisms including N. gonorrhoea. Acinetobacter spp,, Salmonella spp, E.coli and Gardenerella vaginalis. Kellogg and colleagues ${ }^{21}$ tested Chlamydiazyme and the blocking antibody reagent with clinical isolates of bacteria and yeasts and found that the blocking of antibody eliminated the false positive results due to large concentration of some strains of gram negative bacteria. Much higher incidence $(37 \%, 25.8 \%)$ was reported in a study group, which comprised of high-risk population. ${ }^{13,15}$ Low incidence (13.2\%) reported by Grossman ${ }^{16}$ from a high risk population of pregnant women is reported to be due to the increased transportation time (4 days) that was taken before the samples were processed and this had led to a loss of infectious particles/antigens.

The sensitivity of EIA procedure has been reported to range from 44.4 to $100 \%$ for cervical specimens. The sensitivity of the $C$. trachomatis antigen assay is directly related to the number of Chlamydia present in the specimen.

Asymptomatic carriage of $13.6 \%$ reported by us is a cause of concern. Although most infected women were asymptomatic, it has been reported earlier that at least half of infected people are carriers. ${ }^{17}$ The age of peak incidence is late teens and early twenties, which is sexually the most active age.

Genital infections caused by Chlamydia have been documented to be associated with abortions, whether spontaneous or medically terminated pregnancy's. ${ }^{22-27} \mathrm{We}$ report $27.7 \%$ positivity in our study. It is this group that forms a high risk population and requires screening to avoid post abortal complications like PID, ectopic pregnancy and secondary infertility. Yashodhara ${ }^{22}$ reports a very high incidence of $38.5 \%$ in her study, which was based on detection of $\operatorname{IgM}$ and $\mathrm{IgG}$ antibodies. Women experiencing recurrent spontaneous abortions have high titres of anti chlamydial IgG but negative endocervical cultures for $C$. trachomatis. ${ }^{26}$

EIA technique for detection of Chlamydia antigen provides another alternative to culture. The reported sensitivity and specificity of these tests for genital infections (as compared to culture) have been $60-80 \%$ and $97-99 \%$ respectively in high risk populations. EIA offers the potential advantage over direct fluorescence assay (DFA) of objectivity and ease of mechanisation. It also allows batch processing, which is more conclusive in large scale screening. The best EIAs seem to have sensitivity similar to that of DFA in expert hands. However, non-culture tests are subject to false positive results. They should therefore be used with caution in low prevalence settings. Consequently, the interpretation of a positive test result must be handled with care and verification may be desirable. Verification of a positive test can be by either culture or a non culture test that identifies a different Chlamydia antigen, or nucleic acid sequencing or a blocking antibody or, a competitive probe.

Cell culture technique for isolation of $C$. trachomatis are available mostly in large medical centers. The disadvantage of this being, limited availability, low and variable level of sensitivity $(60-80 \%)$, its requirement for vigorous transport conditions and its high cost and technically demanding nature.

Assays using nucleic acid probes like ligase chain reaction (LCR) and polymerase chain reaction (PCR) are also being used, which provides approximately equal results to the best of EIAs in terms of sensitivity and specificity. These tests are now the most sensitive diagnostic methods available for detecting Chlamydia infection, being the first 
non culture assay actually to surpass culture in sensitivity. The ability of these tests to detect Chlamydial genes in urine with high degree of sensitivity and specificity allows their use with urine specimens rather than the conventional urethral and cervical swabs. The use of urine sample is particularly appealing for Public-Health Chlamydia Screening Programmes. However, these tests require trained manpower, expensive equipment and perfect infrastructure; they are expensive to be used for screening purposes in developing countries like ours.

In conclusion, the present study gives us an insight into the overwhelming problem of $C$. trachomatis infection. It is required that all the high risk pregnant women (previous history of abortions) are screened by this simple and reliable antigen detection test using ELISA technique and the positive result be verified by antibody blocking assay. The two methods in conjunction give results comparable to other methods used for diagnosis.

\section{References}

1. McGregor JA and French JI. Chlamydia trachomatis infection during pregnancy. Am J Obstet Gynaecol 1991;164:82-9.

2. Harrison HR, Alexander ER, Weinstein L, Lewis M, Nash M, Sim DA. Cervical C. trachomatis and Mycoplasmal infections in pregnancy. JAMA 1983;250:1721-7.

3. Martin DH, Koutsky L, Eshenbach DA, Dalwig JR, Alexander RE, Benedict JK Prematurity and perinatal mortality in pregnancy complicated by maternal $C$. trachomatis infections. JAMA 1982;247:1585-8.

4. Sweet RL, Landers DV, Warker C, Schachter J. C. trachomatis infection and pregnancy outcome. Am J Obstet Gynaecol 1987;156:824-33.

5. Beaiyan G, Williams I. Prevalence of C. trachomatis Infection in Pregnant women in Zaire. GUM 1990;66:124.

6. Jain A, Nag VL, Goel MM, Chandrawati and Chaturwedi UC. Adverse fetal outcome in specific IgM Positive C. trachomatis Infection in Pregnancy. Indian J Med Res 1991;90:420-3.

7. Schachter J, Grossman M, Holt J, Sweet R, Goodner E, Mills J. Prospective study of Chlamydia infection in neonates. Lancet (ii) $1979 ; 377$.

8. Hammerschlag MR, Chandler JW, Alexander ER, English M, Chiang WT, Koutsky L, et al. Erythromycin ointment for ocular prophylaxix of neonatal Chlamydial infection. JAMA 1980;244:2291.

9. Hammerschlag MR, Andreka M, Semine DZ, McComb D, McCormack WM. Prospective study of maternal and infantile infection with C.trachomatis. Ped 1979;64:142.

10. Schachter J. Chlamydial infection. N Engl J Med 1978;298:540.

11. Joshi JV, Palayekar S, Hazari KT, Shah RS, Chitlange SM. The prevalence of C.trachomatis infection in young women. Natl Med J India 1994;7:57-9.

12. Wood PL, Hobson D, Rees E. Genital infection with C.trachomatis in women attending an Antenatal Clinic. $B J$ Obstet Gynaecol 1984;91:1171.

13. Hardy PM, Hardy JB, Nell EE, Graham DA, Spence MR, Rosenbaum RC. Prevalence of 6 STD agents among pregnant Innercity adolescents and pregnancy outcome. Lancet 1984;11:337.

14. Khurana CM, Deddish PA, delMundo F. Prevalence of $C$. trachomatis in pregnant cervix. Obstet Gynaecol 1985;66:241.

15. Baslekhi VS, McNeeley SG, Ryan G, Robinson M. A comparison on non-culture dependant methods for detection of C. trachomatis infection in pregnant women. Obstet Gynaecol 1987;70:47.

16. John H, Grossman II, Michel E Rivlin, Harrison JC. Diagnosis of Chlamydial infection in pregnant women using the Testpack Chlamydia Diagnostic kit. Obstet Gynaecol 1991;77:801.

17. Mardh PA. Is Europe ready for STD screening. GUM 1997;73:96.

18. Levy RA, Warford AL. Evaluation of the modified Chlamydiazyme Immunoassay for the detection of Chlamydial antigen. Am J Clin Pathol 1986;86:330.

19. Ridgway GL, Oriel JD, Mumtaz G, Mellars B. Comparisons of methods for detecting $C$. trachomatis. J Clin Pathol 1986;39:232.

20. Antonio J, Amortegui MD, Michael P, Meyer MS. EIA for detection of C. trachomatis from cervix. Obstet Gynaecol 1985;65:523.

21. Kellogg JA, Seiple JW, Hick ME. Cross reaction of clinical isolates of bacteria and yeasts with the Chlamydiazyme test for Chlamydia antigen before and after use of blocking reagent. Am J Clin Pathol 1992;97:309.

22. Yashodhara P, Ramalakshmi BA, Raman L, Naidu AN. C. trachomatis serelogy in abortion. Indian J Med Microbiol 1998;16:69.

23. Rao S, Kumar P, Narayan, Shivanda PG. Chlamydia trachomatis antigen detection from cervix by ELISA in women with recurrent abortions. J Obstet Gynacol India 1997:164.

24. Gogate A, Deodar LP, Shah PK, Vaidya D. Detection of C.trachomtis antigen and toxoplasma IgM and Mycoplasma hominis (IgG) antibody by ELISA in women with $\mathrm{BOH}$. Indian J Med Res 1997;100:19.

25. Quinn PA, Petric M, Barkin M, Butany J, Derzko C, Gysla M et al. Prevalence of antibody to C.trachomatis in spontaneous abortions and infertility. Am J Obstet Gynacol 1987;156:291-6.

26. Witkin SS, Ledger WJ. Antibodies to Chlamydia trachomatis in sera of women with recurrent spontaneous abortions. Am J Obstet Gynaecol 1992;167:135-9. 